MATEC Web of Conferences 25,03016

(2015)

DOI: $10.1051 /$ matecconf/ 20152503016

(C) Owned by the authors, published by EDP Sciences, 2015

\title{
One Low-cost Quartz Lamp Radiation Aerodynamic Heating Simulation Experiment System with Control Law Flexible Adjustment Feature
}

\author{
Decheng Wang* \\ Automatic College, Northwest Polytechnical University, Xi'an, Shaanxi, China \\ Qiang Qin \\ China Aircraft Strength Institute, Xi'an, Shaanxi, China \\ Qikang Ren \\ Automatic College, Northwest Polytechnical University, Xi'an, Shaanxi, China
}

\begin{abstract}
The quartz lamp radiation aerodynamic heating simulation experiment system plays an important role on the structure strength heat experiment. In order to reduce its price and enhance flexibility on control law design of experiment system, a design method for low-cost quartz lamp radiation aerodynamic heating simulation experiment system with control law flexible adjustment feature is proposed. The hardware part is constructed by taking Digital Signal Processor (DSP) as an implementing agency controller. The feedback temperature after processed is computed by DSP. But the experiment process control value is computed by computer. The feedback temperature and experiment process control value data are transferred by serial communication model between DSP and computer. The experiment process relation data is saved by computer with EXCEL file, including the given target spectrum, the feedback temperature and the control value. The results of experiments on system identification, PID spectrum tracking, different zone control and the open loop control show the effectiveness of the proposed method.
\end{abstract}

Keywords: aerodynamic heating; quartz lamp radiation; temperature control; DSP

\section{INTRODUCTION}

Aerodynamic heating problem can bring many disadvantages to aircraft, such as structure stiffness reduction, intensity weaken, thermal stress, thermal strain and material ablation, especially for hypersonic flight condition. It must be considered in the design of aircraft structure strength ${ }^{[1-3]}$. There are some kinds of methods for simulating aerodynamic heating, such as convective heating, radiation heating and conduction heating ${ }^{[4-6]}$. As the radiation heating source, quartz lamp has following advantages such as the easier operation, lower expense, easier control for different zones, and the copy shape heater designed with test specimen. These advantages make quartz lamp become the most commonly-used heating source in aerodynamic heating simulation experiment instead of other heating ways.

Some design methods for quartz lamp radiation aerodynamic heating simulation experiment system have been proposed. Those methods can be divided into two kinds. The first kind of method is based on Programmable Logic Controller (PLC) ${ }^{[7,8]}$. The close-loop control function and the feedback data acquisition are carried out by PLC and the price of PLC is very expensive. In the actual experiment process, the controller is far away from test specimen. The thermocouple output signal is overlaid disturb- ance noise due to long distance transmission and space disturbance produced power switching device. The overlaid disturbance noise can reduce feedback signal measurement accuracy. Thus it can affect the control performance and the data communication speed can limit the control period. The second kind of method is based on I/O card and computer ${ }^{[9,10]}$. The control function is carried out by computer. The feedback data acquisition is carried out by $\mathrm{I} / \mathrm{O}$ card. The data communication speed problem is avoided, but the small signal transfer disturbance noise problem still exists. This kind of experiment system is also expensive due to high price of $\mathrm{I} / \mathrm{O}$ card. The feedback temperature channel number is limited, because the input channel of $\mathrm{I} / \mathrm{O}$ card is few. The design of low-cost quartz lamp radiation aerodynamic heating simulation experiment has good demand. Because the quartz lamp radiation aerodynamic heating simulation experiment system has great nonlinear features, it is difficult to get the good spectrum tracking result. Much time is used to debug spectrum tracking. The data storage ability is very important for good spectrum tracking.

Aiming to the low-cost quartz lamp radiation aerodynamic heating simulation experiment system design and enhance the data storage ability, this paper proposed an experiment system design method based on DSP and computer. The experiment result shows that the proposed control method has good performance,

*Corresponding author: wangdecheng@nwpu.edu.cn

This is an Open Access article distributed under the terms of the Creative Commons Attribution License 4.0, which permits unrestricted use, distribution, and reproduction in any medium, provided the original work is properly cited. 


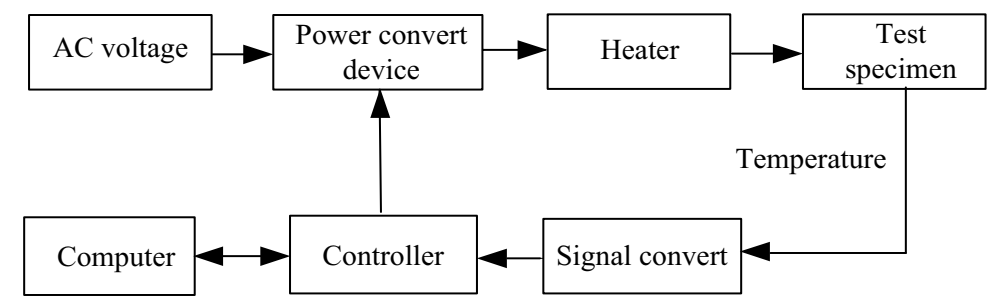

Figure 1. Hardware structure schematic diagram

improving the effectiveness of the aerodynamic heating simulation experiment.

\section{HARDWARE DESIGN OF EXPERIMENT SYSTEM}

The hardware structure of the proposed quartz lamp radiation aerodynamic heating simulation experiment system is shown in Figure 1.

The AC voltage part provides the original energy to experiment system. It directly adopts three-phase AC $380 \mathrm{~V}$ provided by power supply system.

The function of the power convert device part is to dynamically adjust the quartz lamp input voltage, providing the basis for heat condition close-loop control. It is constructed by single phase AC regulating voltage module. The model number is LTVDH-380V $-120 \mathrm{~A}$. This module is controlled by analog voltage signal with range scope from $0 \mathrm{~V}$ to $5 \mathrm{~V}$. This analog voltage signal has a certain relationship between the input voltage and the output voltage of quartz lamp. As the input control voltage changing, the quartz lamp input voltage is changed. There is electrical isolation feature between the control signal and the high voltage signal, enhancing the system safety performance.

The heater is constructed by a series of quartz lamps. The input voltage of each quartz lamp can be $380 \mathrm{~V}$. In order to improve the heat flux output ability, reflectance coating on the surface of quartz lamp is adopted.

The test specimen depends on the experiment object. Different experiments may have different test specimens. Its temperature change is controlled by quartz lamp radiation heat transfer, and its temperature is measured by thermocouple. In our following experiment, the material of the test specimen is titanium alloy with TB type. The K-type thermocouple is used.

The signal convert part is used to convert small voltage signal of thermocouple into big voltage meeting the requirement of following-up part. This part can be placed near the test specimen. The noise produced by long distance transmission and power switching device space disturbance can be solved effectively. By amplified this small signal, the controller can carry out more accurate acquisition of feedback signal. The model number of signal convert part is DS-22-111AD. Its working power source is $24 \mathrm{~V}$.

The function of the controller part is different from traditional controller with auto-computing feature of control value. Its function has three parts: the feedback temperature acquisition, the control vale output and the data communication. It is constructed by DSP with TMS320F2812. In order to achieve corresponding function, the DSP minimum system is constructed. It includes following parts such as power supply part, JTAG part, crystal oscillator part and digital level changing part. The power supply part mainly provides $3.3 \mathrm{~V}$ and $1.9 \mathrm{~V}$ by $5 \mathrm{~V}$ input power supply with special power supply chip. The JTAG part is used to debug the program by online model. After finished the program debugging, the program is programmed in flash memory. The frequency of crystal oscillator is $30 \mathrm{MHz}$. Digital level changing part mainly achieves the digital voltage change which is between $5 \mathrm{~V}$ and $3.3 \mathrm{~V}$. In order to get feedback of temperature, it will acquire the voltage signal using inner analog to digital converter's peripheral function. Sixteen channels' signals can be connected to DSP at the same time. The controller and the computer build the communication relationship by serial communication. The controller sends temperature information to computer. It also receives control value by this digital communication model. In order to achieve digital level changing, the specific communication application chip max3232 is used. What's more, it outputs analog voltage to change output of the power convert device. Because TMS320F2812 inner does not have digital to analog converter function in peripheral, the implement of digital to analog converter function depends on circuit design. Taken into consideration of concise circuit design, the serial digital to analog converter module is adopted. The corresponding analog converter device module number is TLC 5615 .

The function of computer includes following parts, such as control vale computing, human-computer interaction, data communication and data storage. By serial communication, the computer can get feedback temperature and send control vale to DSP. The implement of human-computer interaction function depends on the software design. By human-computer interaction, the operator can design the experiment target spectrum. The experiment process condition can be monitored and the control law parameters can be set freely. By control law and feedback temperature information, it produces new control value. The experiment process data is saved in EXCEL file, including the target spectrum data, the feedback temperature data and the control value data. 
The whole experiment system hardware is shown in Figure 2 and Figure 3. Figure 2 mainly contains the controller part and the signal convert part. The switching power supply is used to provide working power supply with $5 \mathrm{~V}$ and $24 \mathrm{~V}$. Figure 3 mainly contains the heater part, the power convert device part and the test specimen part. The breaker is used to improve the system safety performance.

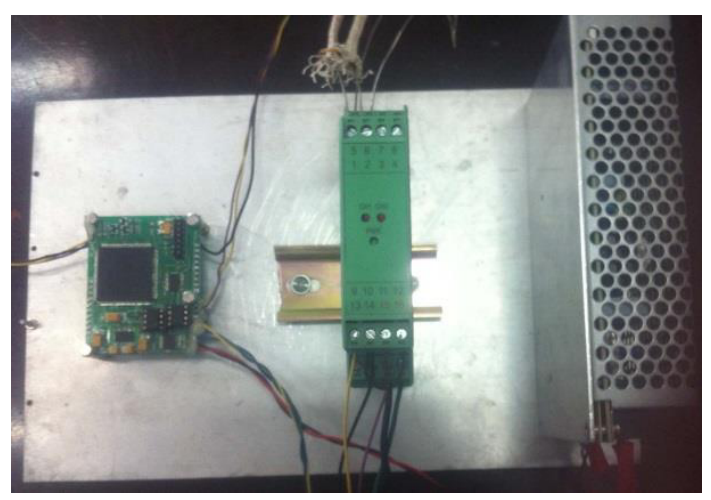

Figure 2. The controller part and the signal convert part

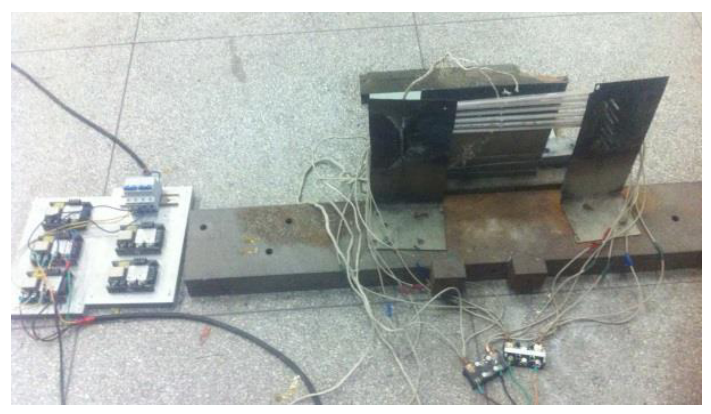

Figure 3. The heater part, the power converter device part and the test specimen part

\section{SOFTWARE DESIGN OF EXPERIMENT SYS- TEM}

The software design is divided into two parts, including the controller software part and the computer software part.

The controller software part is carried out by TMS320F2812. It is developed by Code Composer Studio (CCS) with $\mathrm{C}$ language. The corresponding program function can be divided into five parts: data acceptance, data transmission, analog signal output, sampling and filtering. The data acceptance part is implemented by interrupting way. When the acceptance interrupt happened, the data saved in the corresponding receiving data buffer register is assigned to the variable. The data transmission part is implemented by inquiring way. By equal time interval, the data will be written into the corresponding transmission data buffer register, as buffer register empty.
The buffer register data is sent to computer by serial model under the baud rate control. In analog signal output part, the serial clock signal and the enable signal are provided by digital output signal with input/output function of peripheral. In sampling part, the timer is used to realize equal internal sampling. The filtering part is used to reduce the noise influence on feedback temperature accuracy measurement.

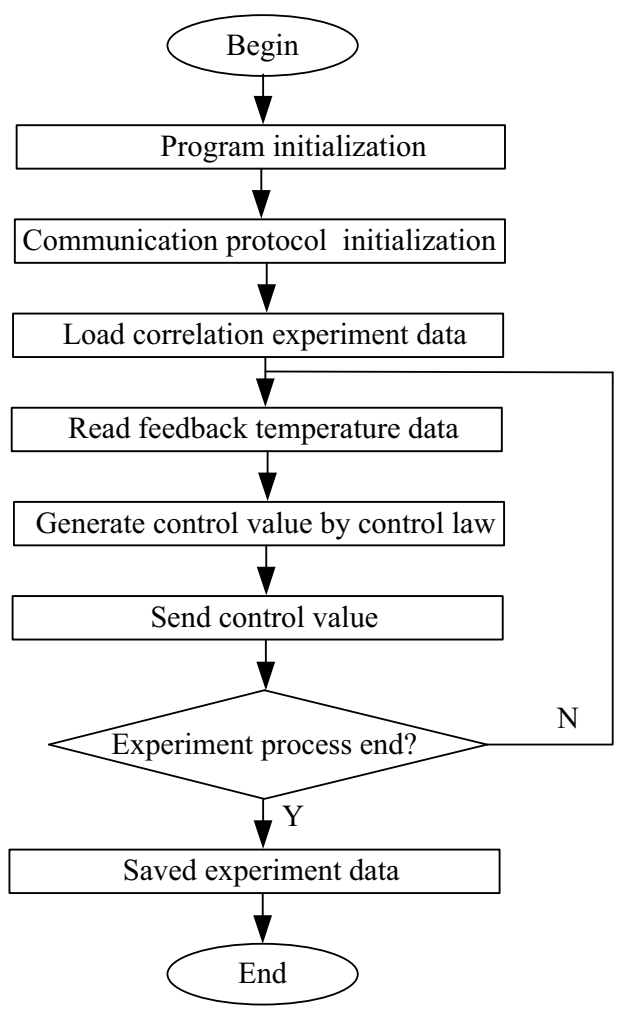

Figure 4. Computer software flow chart

The computer software part is carried out by computer. It is developed by LabWindows/CVI based on $\mathrm{C}$ language. It mainly provides the human-computer interaction interface. The corresponding program function can be divided into five parts: data acceptance, data transmission, data saving, control value computing. The corresponding program flow chart is shown in Figure 4. The experiment correlation data is saved in EXCEL file. Before the beginning of experiment, the target spectrum should be given by EXCEL file style. The aim of communication protocol initialization is to ensure the serial communication reliability. By reading corresponding EXCEL file, the target spectrum data is assigned to the corresponding array. In order to keep the same control period, the timer is adopted. At the beginning of the experiment, the timer is enabled. At the end of the experiment, the timer is shut off. By comparing the feedback temperature and the target spectrum, the new control value is produced based on the corresponding control law. The target 


\section{MATEC Web of Conferences}

spectrum data, feedback temperature data and control value data are saved in different array. Whether the experiment process finished depends on checking the target spectrum data. After the experiment process finished, those experiment process data are saved by writing EXCEL file. At the end of experiment process, the control value is set to zero in order to enhance the experiment safety performance.

\section{EXPERIMENTAL ANALYSIS}

In order to verify effectiveness of the proposed quartz lamp radiation aerodynamic heating simulation experiment system, the flowing experiment is carried out.

It is very important for experiment system with system identification feature. The reason is summarized as the following two aspects. The first one is that the system identification can give the guidance for control law design by traditional parameter adjustment method. The second one is that the system identification provides a basis for experiment system feature analysis. And the first experiment is carried out on system identification. The control signal of power convert device is selected as the system identification of given signal. The given signal is changed with range which is from $1 \mathrm{~V}$ to $3 \mathrm{~V}$ by interval with $0.5 \mathrm{~V}$, and each stage lasts 3 minutes. The feedback temperature is shown in Figure 5. This feedback curve can be used to construct an implementing agency model by mechanism analysis. It can be also used to conduct the control law design.

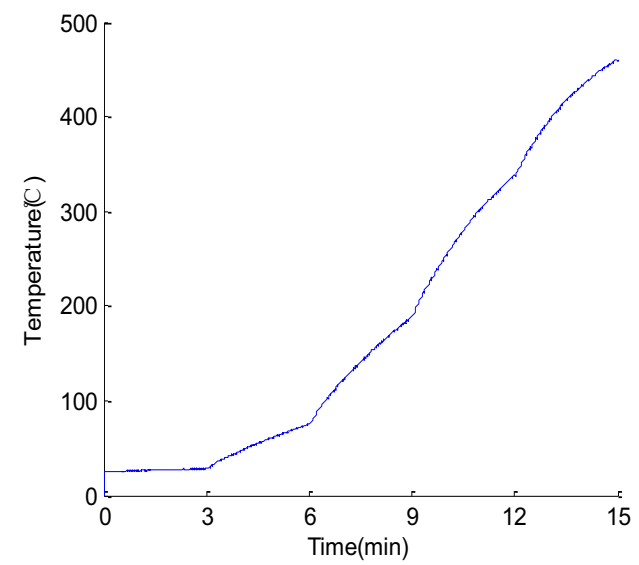

Figure 5. Feedback temperature for system identification

The most commonly-used spectrum tracking debug method is PID. The second experiment is carried out on PID tracking. By the proposed experiment system, PID parameters, including the proportional parameter, the integral parameter and the differential parameter, can be set freely. Figure 6 gives a PID spectrum tracking result based on the system identification re- sult conducting parameters design. The given spectrum curve and the feedback temperature curve are plotted in Figure 6. We cannot recognize these two curves, because the tracking result is very good.

Different positions on weapon may have different thermal characteristics because of flight characteristics. Thus, different places on test specimen may have different target spectrum during experiment process. Different zone control ability is very important for aerodynamic heating simulation experiment system and the third experiment is carried out on different zone control. Figure 7 gives different zone control results. Two different zones are given different target spectrum. The design of controller parameters is also based on the system identification result. The given target spectrum and the feedback temperature curve are plotted in the same figure. We cannot recognize these two curves, because the tracking result is very good.

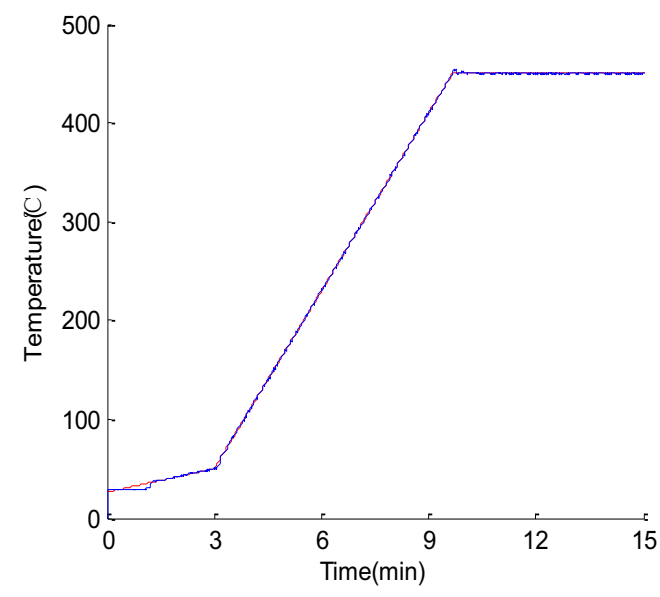

Figure 6. PID spectrum tracking result

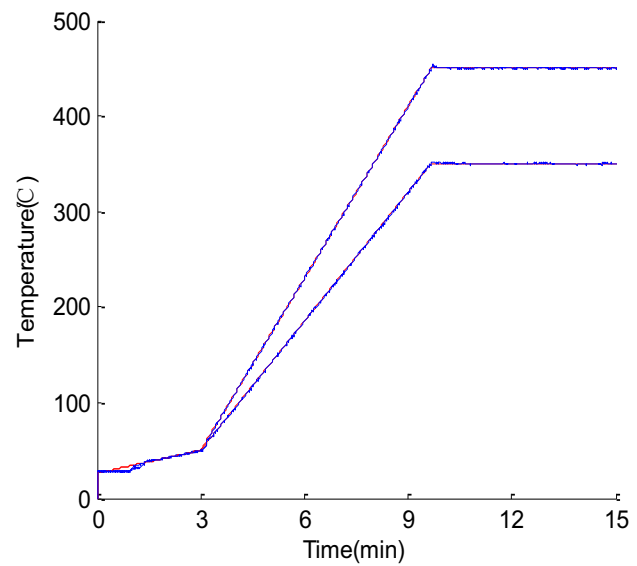

Figure 7. Different zone control results

The given target spectrum used in aerodynamic 
heating simulation experiment has stable feature during the whole experiment process. This feature gives the operator the chance using some new control methods and debugging spectrum tracking, such as repetitive learning method and iterative learning method. When those methods are used, the experiment system should have the control value storage function, because those control values are used to design new control value. The fourth experiment is carried out in order to show the control value storage function of experiment system. The open loop control with control value used in second experiment is shown in Figure 8. It has similar feedback result with Figure 6.

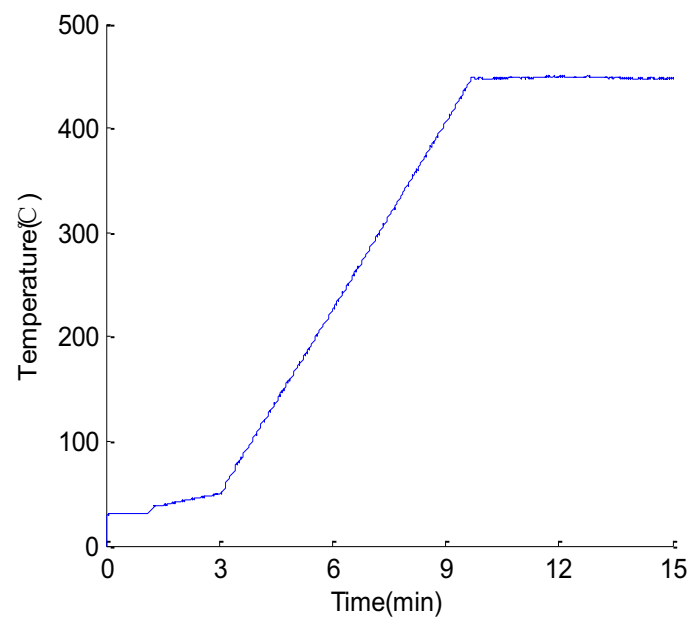

Figure 8. Open loop control result

\section{CONCLUSION}

Aiming at quartz lamp radiation aerodynamic heating simulation experiment design problem, this paper gives an experiment system design method. Compared with traditional method, it is low-cost because of taking DSP as controller. The software design makes the experiment system has following functions, such as system identification, PID tracking, different zone control and control value storage. The experimental analysis result shows the effectiveness of the proposed method based on corresponding experiment analysis. It can provide an effective way for aerodynamic heating simulation experiment.

\section{ACKNOWLEDGEMENT}

The authors would like to acknowledge support from Aeronautical Science Foundation of China (Grant No. 20140953016). And the authors would also like to thank all the reviewers for their precious comments.

\section{REFERENCES}

[1] Xuqi,F. 2009. High speed flight vehicle structure analysis and application, Beijing: national defence industry press.

[2] Chun, S., Fengxian, S., Xinlin, X., Zhanwei,C., Mingxing, Y. \& Zhenfeng, W. 2012. Transient characteristics of aerodynamically heating partial porous wall-Inner cavity.Journal of Astronautics33:1006-1012.

[3] Culler,A.J.,\& McNamara,J.J.2010. Studies on fluid-thermal-structural coupling for aerothermo-elasticity in hypersonic flow. AIAA Journal48:1721-1737.

[4] Peter,H.,Chu,C.,\&Marksberry,C.L.2015.High hypersonic wind tunnels and propulsion test facilities, AIAA-2005-3442.

[5] Holden, M.S., Wadhams, T.P.,\& Candler,G.V.2004. A review of experimental studies in the LENS shock tunnel and expansion tunnel to examine real-gas effects in hypervelocity flows. Proceeding of 42th AIAA Aerospace Sciences Meeting and Exhibit: 916-932.

[6] Dafang, W., Shougen, Z., Bing, P.,Yuewu, W., Meng, M. \& Shuang, W. 2012. Experimental study on thermal-vibration test for wing structure of high-speed cruise missile.ActaAeronauticaetAstronauticaSinica33:1-10.

[7] Decheng, W., Lei, W., Haidong, W. \& Guohao, L. 2015. One Adaptive Control Method used in Quartz Lamp Radiation Aerodynamic Heating Simulation Experiment. Proceedings of 2015 International Conference on Computer and Computational Sciences: 83-86.

[8] Junfei,L.,Hui,L.,\&Pan,L.2012. An aerodynamic heating simulation system based in Matlab. Mechatronics 19:25-27.

[9] Dafang, W., Bing, P., Zhentong, G., Meng, M., Lin, Z. \& Yuewu, W. 2012. On the experimental simulation of ultra-high temperature, high heat flux and nonlinear aerodynamic heating environment and thermo-machanical testing technique. Journal of Experimental Mechanics27:255-271.

[10]Dafang, W., Yunpeng, F.\& Min,Z. 2003. Experimental simulation system of transient aerodynamic heating for high speed flight vehicles. Aviation Metrology \& Measurement Technology23:9-14. 